\title{
The expressions of bHLH gene HES1 and HES5 in advanced ovarian serous adenocarcinomas and their prognostic significance: a retrospective clinical study
}

\author{
Xinyu Wang $\cdot$ Yajuan Fu $\cdot$ Xiaoduan Chen $\cdot$ Jing Ye \\ Bingjian Lü $\cdot$ Feng Ye $\cdot$ Weiguo Lü $\cdot$ Xing Xie
}

Received: 10 September 2009/Accepted: 1 December 2009/Published online: 20 December 2009

(C) The Author(s) 2009. This article is published with open access at Springerlink.com

\begin{abstract}
Purpose Notch signaling was recently found to be associated with prognosis of some cancers. The aim of the study is to investigate significance of the expression of HES1/HES5 protein, downstream effectors of Notch, in prognosis of the patients with advanced ovarian epithelial cancers.

Methods Formalin-fixed, paraffin embedded tissues and clinic-pathological parameters from 61 patients with FIGO stage IIIc-IV ovarian serous adenocarcinoma were collected, the expression of HES1 and HES5 protein were immunohistochemically detected, and the association of HES1 and HES5 expression with survival of the patients were analyzed.

Results The expressions of both HES1 and HES5 in adenocarcinoma were significantly higher than those in adenoma and normal control $\left(\chi^{2}=32.915, P=0.000\right.$ and $\chi^{2}=46.863, P=0.000$ respectively). Overall survival and disease-free period were longer in HES1 low-expression patients (median 43.0 and 22.0 months) than those in high-expression patients (median 24.0 and 14.5 months). Of those, Overall survival period of patients with HES1 low-expression was significantly longer than that of those with high-expression $\left(\chi^{2}=4.049, P=0.044\right)$. Univariate
\end{abstract}

X. Wang and Y. Fu contributed equally to this article.

X. Wang $\cdot$ Y. Fu $\cdot$ J. Ye $\cdot$ F. Ye $\cdot$ W. Lü $\cdot$ X. Xie $(\bowtie)$

Women's Reproductive Health Laboratory of Zhejiang Province, Department of Gynecologic Oncology, Women's Hospital,

School of Medicine, Zhejiang University,

310006 Hangzhou, China

e-mail: xiex@mail.hz.zj.cn

X. Chen · B. Lü

Women's Reproductive Health Laboratory of Zhejiang Province,

Department of Pathology, Women's Hospital,

School of Medicine, Zhejiang University,

310006 Hangzhou, China analysis and multivariate Cox regression model did not show that HES1 or HES5 expression was a factor associated with survival of advanced ovarian serous adenocarcinoma patients.

Conclusions The expressions of bHLH gene HES1 and HES5 are increased in advanced ovarian serous adenocarcinomas, and HES1 high-expression probably is a potential poor prognostic factor for the patients.

Keywords Ovarian serous cancer - bHLH gene · HES gene $\cdot$ Prognosis

\section{Background}

Epithelial ovarian cancer is the leading cause of death from gynecologic cancers, accounting for 5\% of all cancer deaths. Despite of efforts aiming at improving methods of early diagnosis, the majority of cases of ovarian cancer are not diagnosed until the disease has spread throughout whole peritoneal cavity. Since Munnell introduced a concept of the "maximum surgical effort" for ovarian cancer in 1968, cytoreductive surgery as the primary management of ovarian cancer has been widely performed in the world. Meanwhile, a series of novel chemotherapeutic agents and combination drug regimens have been identified to be clinically active. Primary cytoreductive surgery followed by platinum-based combination chemotherapy has been recognized as a standard strategy of management for ovarian cancer. However, there appears to be little change in ovarian cancer mortality in past several decades, and five-year survival rates of those with advanced disease have been still remaining under 30\% (Hoskins et al. 2000).

It is well known that many factors affect ovarian cancer prognosis, such as the age of patient, tumor stage, 
volume of residual disease, histological subtype and grade, preoperative CA-125 level, amount of ascites, and others. Surgery alone is rarely, if ever, curative for patients with advanced ovarian cancer, even in those without gross residual disease after successful cytoreduction. It is necessary for all of the advanced ovarian cancer patients to undergo chemotherapy. Thus, the response to chemotherapy should be the most important factor associated with prognosis of ovarian cancer patients. Various kinds of mechanisms are involved in resistance to chemotherapy, including pharmacokinetics, tumor microenvironment and intrinsic factors in tumor cells. Of those, the latter is a key factor that influences the response to chemotherapy. It has been verified that many signal molecules controlling cellular proliferation, division, differentiation and death participate in resistance to chemotherapeutic drugs in cancer cells, for instance, microtubule-interfering agent (e.g. EM012), protein kinase (e.g. PI3K/Akt), epithelial growth factor receptor, and others (Zhou et al. 2005; Mozzetti et al. 2005; Lee et al. 2005; Park et al. 2005; Fu et al. 2007). Recently, it was found that survival signaling by Notch participated in not only carcinogenesis but also chemoresistance in some malignancies (Nefedova et al. 2004; Mungamuri et al. 2006). However, the role of basic-helixloop-helix transcription factors (bHLH genes) including HES genes, as downstream molecules and effectors of Notch, in regulating the response to chemotherapy for cancer is rarely reported and still poorly understood up to date, though it has been revealed that the expression of HES protein is associated with the prognosis of patient with cervical cancer (Liu et al. 2007), mucoepidermoid carcinoma (Behboudi et al. 2006), and others.

Ovarian serous tumor is the most common histological subtype arising from the surface epithelium of the ovary, and accounts for nearly half of ovarian epithelial tumors, of those, about one-third are malignancies. In the study, we collected the formalin-fixed, paraffin embedded tissues and clinic-pathological parameters from 61 patients with stage IIIc-IV ovarian serous adenocarcinoma, immunohistochemically detected the expressions of HES1 and HES5 protein, and analyzed their associations with survival of the patients. The aim of the study was to investigate significance of HES1/HES5 protein expression in prognosis of the patients with advanced ovarian epithelial cancers.

\section{Materials and methods}

Specimen and patient

All specimens were obtained from the Pathological Department of Women's Hospital, School of Medicine, Zhejiang University from January 1999 to December 2004.
During this period, the Gynecologic Department of the Hospital adopted 461 ovarian cancer patients. In 461 patients, 104 underwent primary cytoreductive surgery in our Department, diagnosed as FIGO stage IIIc-IV diseases, and histologically confirmed as serous adenocarcinoma. Of those, 61 received total six-course platinum-based combination chemotherapy following surgery in our Department and had completely clinic-pathological data. Thus, those 61 patients were finally enrolled into the study. Meanwhile, tissues from 20 normal ovaries and 47 ovarian serous adenomas were collected as controls in our study. Normal ovarian tissues were collected from perimenopausal women with myoma who underwent adnexectomy and hysterectomy. The informed consent on collection of samples was obtained by each patient. The study was approved by the Medical Ethics Committee of Women's Hospital, School of Medicine, Zhejiang University according to the guidelines of in compliance with the Helsinki Declaration.

In 61 ovarian serous adenocarcinoma patients (2474 years, median 49 years) undergoing primary cytoreduction, 28 patients had equal to or less than $2 \mathrm{~cm}$ of residual tumor and 33 patients more than $2 \mathrm{~cm}$; 55 patients were FIGO stage IIIc and 6 patients stage IV; 5 patients were pathological grade $1(\mathrm{G} 1), 19$ patients $\mathrm{G} 2$, and 37 patients G3 according to WHO grading system. All of the patients underwent platinum-based combination chemotherapy for six courses following primary surgery. Chemotherapy regimens included PC (cisplatin/carboplatin and cyclophosphamide) in 25 patients, PAC (cisplatin/ carboplatin, adriamycin, and cyclophosphamide) in 11, TP (paclitaxel and cisplatin) in 12, and TC (paclitaxel and carboplatin) in 13 . The interval of chemotherapy was 28 days and the dose of drug was calculated according to the body surface area or AUC such as carboplatin.

Chemotherapy response was evaluated after six courses of chemotherapy for each patient. The evaluation of chemotherapy response was according to objective response or CA125 response criteria. Objective response was assessed with the WHO criteria (Miller et al. 1981) and CA125 response was evaluated according to the criteria proposed by Bridgewater et al. (1999). Complete and partial remission were regarded as effective and remained ones as noneffective.

All of the patients were followed up by interview in clinic or phone call. The total period of follow-up was 12-108 months.

Clinic-pathologic parameter collection

The clinic-pathologic parameters associated with prognosis of the patients were collected, including the age of patients, pathological grade, size of residual tumor, ascites amount, 
pre-operative maximum tumor diameter, preoperative serum CA125 level, and response to primary chemotherapy.

Immunohistochemistry and evaluation

The rabbit anti-human polyclonal antibodies specific for HES1 and HES5, respectively, were purchased from United States Biological (Swampscott, MA, USA).

All tissues were immediately fixed in $10 \%$ neutralized formalin for $24 \mathrm{~h}$ prior to processing to paraffin wax using standard procedures. Paraffin sections $(4 \mu \mathrm{m})$ were used for histologic diagnosis or immunohistochemistry. Tissue sections were dewaxed and rehydrated using standard procedures. Hydrated autoclave pretreatment was in $10 \mathrm{mM}$ citrate buffer ( $\mathrm{pH}$ 6.0) boiling for $2 \mathrm{~min}$. Endogen peroxidase activity was blocked with $3 \%$ hydrogen peroxidase for $10 \mathrm{~min}$ at room temperature (RT). Slides were incubated with polyclonal rabbit antibodies diluted 1:400 (HES1) and 1:100 (HES5) in Tris-buffered solution (50 mM Tris- $\mathrm{HCl}, 150 \mathrm{mM} \mathrm{NaCl}, \mathrm{pH} 7.4$ ) at RT for $1 \mathrm{~h}$; then incubated with Dako Envision ${ }^{\mathrm{TM}}$ Peroxidase (Dako Diagnostica, Hamburg, Germany) for $30 \mathrm{~min}$ at RT. For visualization of the antigen, 3,3'-diaminobenzidine tetrahydrochloride (Dako) was added at RT. Slides were washed $3 \times 5 \mathrm{~min}$ in phosphate buffered saline (PBS)Tween between each step. Then the slides were counterstained with Mayer's hematoxylin, rinsed in tap water, dehydrated, placed in xylene, and mounted. Blank controls were performed by replacing primary antibodies with normal rabbit serum (Dingguo, Beijing, China). Positive cell was indicated by the presence of distinct brown in the nuclear or cytoplasm of cells. The number of positively stained cells out of 100 in ten random fields $(400 \times$ objective) was counted and represented as the percentage of positive cells. The semiquantitative immunoreactive score was ranged from $-(0)$ to +++ (3) based on the percentage of positive cells and the stain intensity. The percentage of positive cells was scored as follows, - (0) means less than $5 \%$ positive cells; + (1) $5-25 \%$ positive cells; ++ (2) $26-75 \%$ positive cells; and +++ (3) more than $76 \%$ positive cells. The staining intensity was also scored on a four-tiered scale (negative 0, low intensity positive staining 1 , moderate intensity positive staining 2 , and strong intensity positive staining 3). The staining intensity score adding the positive cell score is the overall score. Slides, as well as IHC scorings, were independently reviewed by two empirical pathologists (two of authors) without the knowledge of clinical data.

In order to perform a sensitive statistical analysis, all cases were divided into two groups according to HES1/ HES5 expression. Significant statistical association with prognosis was observed using a cut-off of four in the overall score of HES1/HES5 expression. Patients with overall score $\leq 4$ were called low-expression, while patients with overall score $>4$ were called high-expression (Innocenzi et al. 2003).

Statistics

Chi-square test was performed using SPSS 16.0 software package for Windows. Multivariate Cox regression model was performed to identify independent risk factors associated with patient survival. A level of 0.05 was chosen to indicate statistical significance. All reported $P$ values were bilateral.

\section{Results}

HES1 and HES5 expressions in ovarian serous adenocarcinoma

HES1 and HES5 immunostaining were located in cellular nucleus or cytoplasm. Both HES1 and HES5 were moderately or strongly expressed in ovarian serous carcinoma, in contrast to weakly or not expressed in adenoma and normal ovarian tissues. Typical HES1 and HES5 immunostaining in normal, serous adenoma, and carcinoma were shown in Fig. 1.

There were significant differences of HES1 and HES5 expression among normal, adenoma, and carcinoma groups $\left(\chi^{2}=32.915, P=0.000\right.$ and $\chi^{2}=46.863, \quad P=0.000$ respectively). Expressions of HES1 and HES5 in carcinoma were significantly higher than those in adenoma and normal control, but not different between adenoma and normal (Table 1). The expression between HES1 and HES5 was significantly correlated in ovarian serous adenocarcinoma ( $r=0.284, P=0.027)$.

The associations of HES1 and HES5 expression with the response to chemotherapy for stage IIIc-IV ovarian serous adenocarcinoma

In 61 patients, 40 were effective and 21 non-effective to chemotherapy. There were no significant associations of HES1 and HES5 expression with chemotherapy response (Table 2).

The associations of HES1 and HES5 expression with the prognosis of ovarian serous adenocarcinoma patients

All 61 patients were followed up for 12-108 months, of those, 11 patients were lost. In remained 50 patients, 39 patients relapsed and seven underwent second operation, 11 did not relapse till end of follow-up. Median overall and 
Fig. 1 Expressions and localizations of HES1 and HES5 protein in normal ovarian tissue, adenoma and adenocarcinoma. The distinct brown staining was located in the nuclear or cytoplasm of positive cells

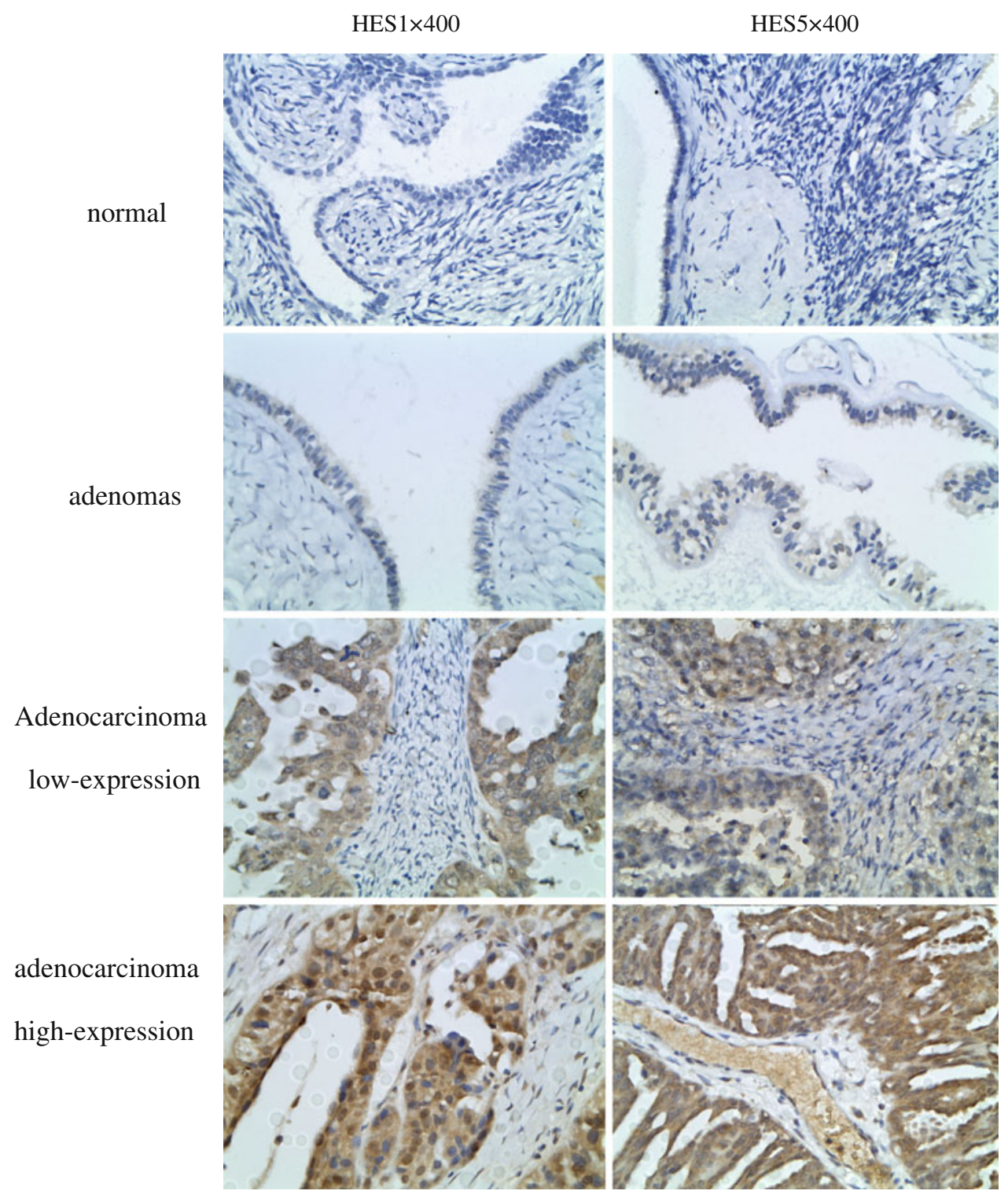

Table 1 Expressions of HES1 and HES5 in different ovarian tissues

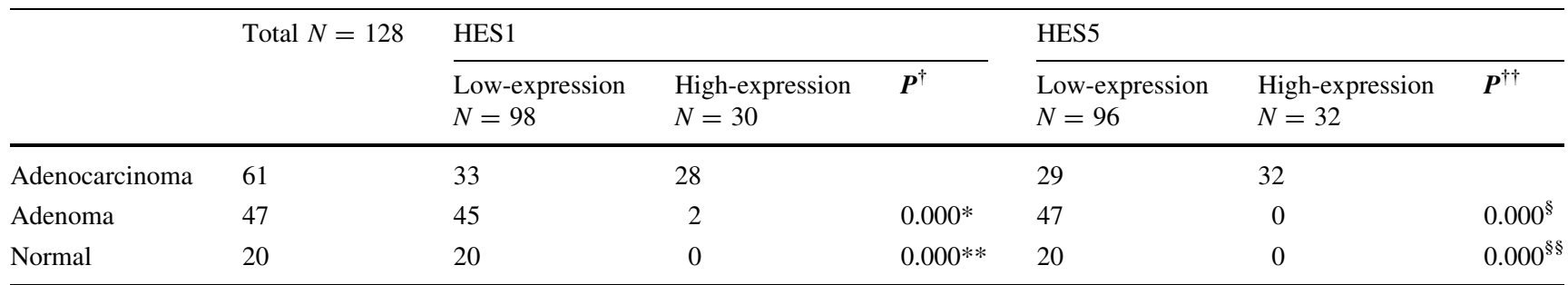

${ }^{\dagger} P$ value among three groups in HES1, $\chi^{2}=32.915, P=0.000$

it $P$ value among three groups in HES5, $\chi^{2}=46.863, P=0.000$

* Adenomas versus adenocarcinoma, $\chi^{2}=22.95$

** Normal versus adenocarcinoma, $\chi^{2}=14.030$

$\S$ Adenomas versus adenocarcinoma, $\chi^{2}=35.037$

$\S \S$ Normal versus adenocarcinoma, $\chi^{2}=17.344$ 
Table 2 The associations of Hes1 and Hes5 expression with the response to chemotherapy for stage IIIc-IV ovarian serous adenocarcinoma

\begin{tabular}{|c|c|c|c|c|c|c|c|c|c|}
\hline & \multirow[t]{2}{*}{ Total $N=61$} & \multicolumn{4}{|l|}{ HES1 } & \multicolumn{4}{|l|}{ HES5 } \\
\hline & & Low-expression & High-expression & $\chi^{2}$ & $P$ & Low-expression & High-expression & $\chi^{2}$ & $P$ \\
\hline Chemo-response & & & & 0.038 & 0.845 & & & 1.146 & 0.284 \\
\hline Non-effective & 21 & 11 & 10 & & & 8 & 13 & & \\
\hline Effective & 40 & 22 & 18 & & & 21 & 19 & & \\
\hline
\end{tabular}

disease-free survival period for the patients with HES1 low-expressions were 43.0 and 22.0 months, while those with high-expressions were only 24.0 and 14.5 months respectively. Overall survival period in patients with HES1 low-expression was significantly longer than that in those with high-expression $\left(\chi^{2}=4.049, P=0.044\right)$, but no differences of overall and disease-free survival periods were found between patients with HES5 low-expression and high-expression, as shown in Fig. 2 and 3. Both univariate analysis and multivariate Cox regression model did not show that HES1 or HES5 expression was a factor associated with survival of advanced ovarian serous adenocarcinoma patients, as shown in Tables 3 and 4.

\section{Discussion}

Mammary cell fate and cell differentiation are governed by a variety of signaling pathways in which Notch signaling cascade plays a key role. In many settings, cell fate decision occurs through ligand-receptor interactions that utilize Notch signaling cascade. Receptor activation by a Notch ligand can lead to the specific fate of a cell, while failure of activation leads to a different fate (Politi et al. 2004). Helix-loop-helix (bHLH) genes are important targets in mammalian Notch pathway. HES gene, homolog of Drosophila hairy and enhancer of split, is one member of the bHLH superfamily of DNA binding transcription factors and expressed in a wide variety of cells, particularly in epithelial and neuro-epithelial cells of the developing embryo (Axelson 2004). There are seven subtypes in the HES gene, including HES1 throughout HES7. Among seven members, HES1 and HES5 are essential effectors of Notch signaling, the expression of which is up regulated by Notch activation. As mediators of Notch signaling, HES1 and HES5 normally have a key role in the process of embryogenesis or in neural stem cells (Axelson 2004; Shi et al. 2008). Mis-expressions of HES1 and HES5 inhibit neuronal differentiation and maintain neural stem cells in the embryonic brain, in contrast, neural progenitors undergo premature neuronal differentiation in HES1 and HES5 double knockout mice (Shi et al. 2008). In addition to CNS, recent studies have revealed that HES1 and HES5 also participate in the regulation of differentiation in other
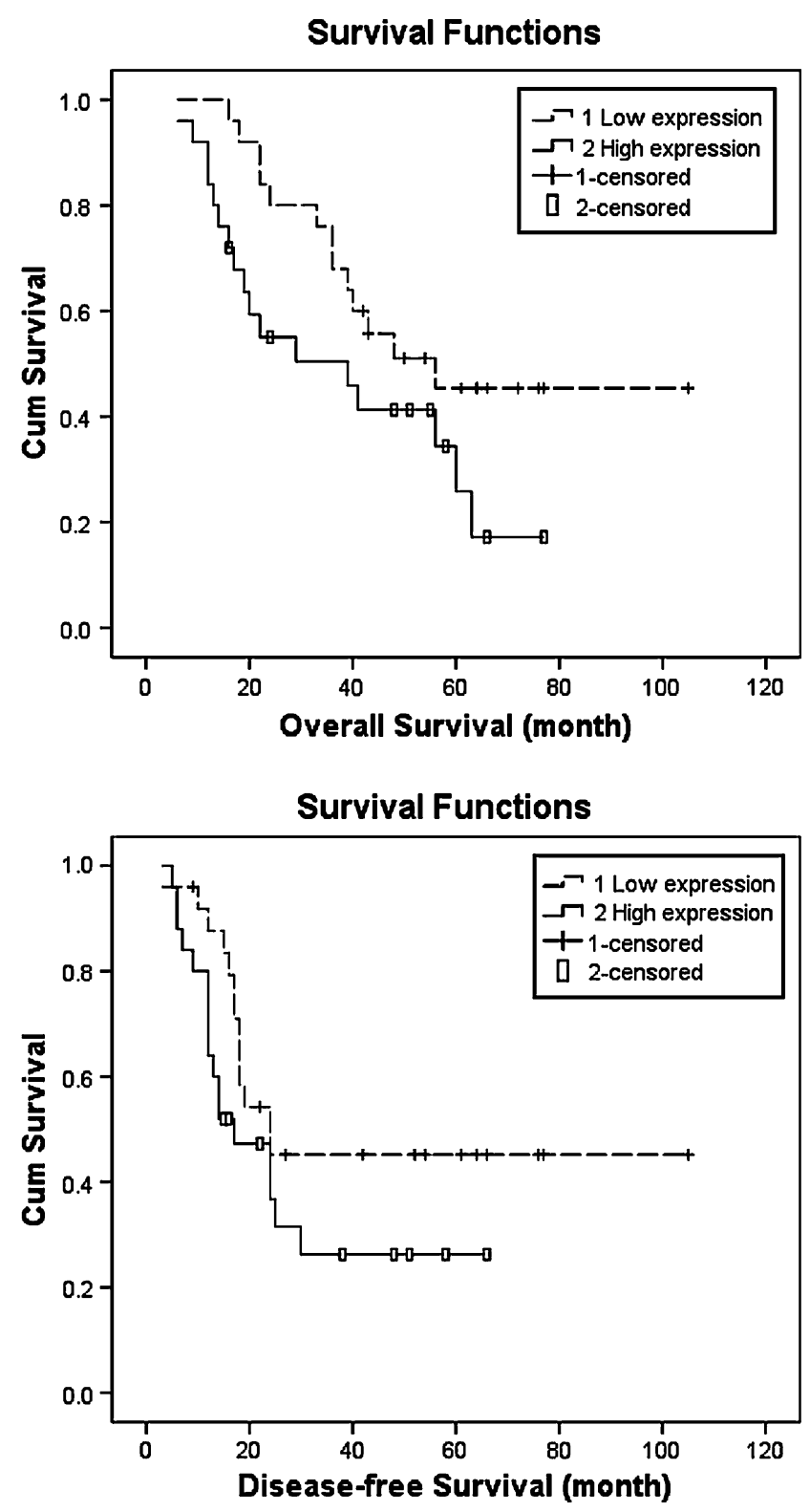

Fig. 2 Overall and disease-free survivals of the patients with HES1 low-expression and high-expression. Overall survival period of patients with HES1 low-expression was significantly longer than that of those with high-expression $\left(\chi^{2}=4.049, P=0.044\right)$

tissue and cell types, such as blood cells, endocrineexocrine cells, somites, adipocytes, muscles, and so on (Miyazaki et al. 2005; Ross et al. 2006). HES genes are 

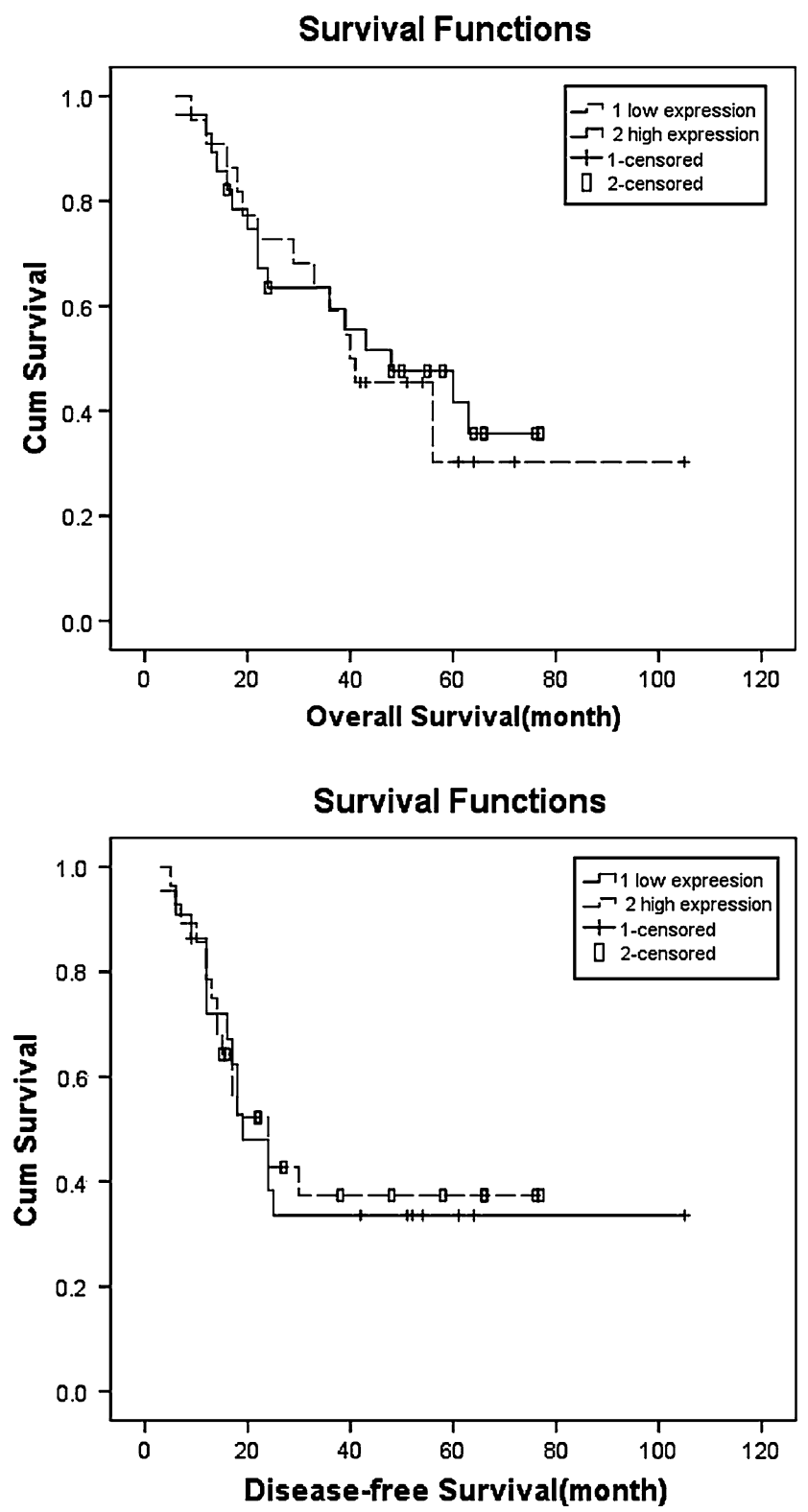

Fig. 3 Overall and disease-free survivals of the patients with HES5 low-expression and high-expression. No differences of overall or disease-free survival between HES5 low-expression and high-expression were found

thought to prevent cellular differentiation and maintain the population of undifferentiated precursor cells (Jensen et al. 2000). Thus, the function of HES genes implies that they may involve in the occurrence and development of tumors. Recent studies have shown mis-expressions of HES1 and HES5 in human malignancies such as lung cancer, cholangiocarcinoma, murine leukemia, and others (Ishimura et al. 2005; Ito et al. 2000), but some of the results appeared to oppose to their function in physiologic condition. For instance, activation of HES1 expression induced the differentiation of neuroblastoma cells, the process of
Table 3 Univariate analysis on the associations of HES1/HES5 expression and clinic-pathological parameters with 5-year survival rate

\begin{tabular}{|c|c|c|c|c|}
\hline $\begin{array}{l}\text { Clinic-pathological } \\
\text { parameter }\end{array}$ & No. & $\begin{array}{l}5 \text {-year } \\
\text { survival } \\
\text { rate }(\%)\end{array}$ & $\chi^{2}$ & $P$ value \\
\hline \multicolumn{5}{|l|}{ Age (year) } \\
\hline$\leq 50$ & 32 & 21.9 & 0.015 & 0.901 \\
\hline$>50$ & 18 & 27.9 & & \\
\hline \multicolumn{5}{|l|}{ Pathological grade } \\
\hline G1 & 4 & 0.0 & 0.918 & 0.764 \\
\hline G2 & 15 & 26.7 & & \\
\hline G3 & 31 & 22.6 & & \\
\hline \multicolumn{5}{|c|}{ Maximum tumor diameter $(\mathrm{cm})$} \\
\hline$\leq 10$ & 36 & 22.2 & 0.011 & 0.918 \\
\hline$>10$ & 14 & 28.6 & & \\
\hline \multicolumn{5}{|l|}{ Ascites amount (ml) } \\
\hline$\leq 500$ & 27 & 29.6 & 1.020 & 0.313 \\
\hline$>500$ & 23 & 17.4 & & \\
\hline \multicolumn{5}{|c|}{ Size of residual tumor $(\mathrm{cm})$} \\
\hline$\leq 2$ & 22 & 40.9 & 6.158 & 0.013 \\
\hline$>2$ & 28 & 10.7 & & \\
\hline \multicolumn{5}{|l|}{ Serum CA125 (u/ml) } \\
\hline$\leq 1,000$ & 27 & 29.6 & 1.020 & 0.313 \\
\hline$>1,000$ & 23 & 17.4 & & \\
\hline \multicolumn{5}{|l|}{ HES1 } \\
\hline Low-expression & 25 & 32.0 & 1.754 & 0.185 \\
\hline High-expression & 25 & 16.0 & & \\
\hline \multicolumn{5}{|l|}{ HES5 } \\
\hline Low-expression & 22 & 18.2 & 0.729 & 0.393 \\
\hline High-expression & 28 & 28.6 & & \\
\hline
\end{tabular}

which was probably inhibited by Notch1 (Axelson 2004), induction of HES1 led to suppression of proliferation in carcinoid tumor cells (Kunnimalaiyaan et al. 2005) and HES1 could inhibit $17 \beta$-estradiol-induced cell proliferation in breast cancer (Ström et al. 2000). However, the recent studies also revealed opposite results. Hopfer and colleagues reported that HES1 protein was strongly expressed in 18/19 ovarian epithelial carcinoma samples (Hopfer et al. 2005). Kimura et al. (2007) recently showed via establishing pancreatic cancer in mice that Notch1 and HES1 were immunohistologically expressed in lesions ranging from tubular complexes to carcinoma in tHESe chemically induced pancreatic tumors. Disagreed results regarding HES effect on tumorigenesis reported by different authors may be due to different cancer cell types. A recent study of ours showed that not only HES1 and HES5 expression were significantly higher in cervical carcinoma compared with cervical intraepithelial neoplasia and normal cervical epithelium, but also over-expressions of HES1 and HES5 were positively correlated with some poor 
Table 4 Cox regression model analysis on the association of HES1/HES5 expression and clinic-pathological factors with 5-year survival rate

Partial regression coefficient

b Standard error

c Hazard ratio

\begin{tabular}{lrlll}
\hline Variable & \multicolumn{1}{c}{$\mathrm{B}^{\mathrm{a}}$} & $\mathrm{SE}^{\mathrm{b}}$ & $\mathrm{HR}^{\mathrm{c}}(95 \% \mathrm{CI})$ & $P$ value \\
\hline Age & -0.344 & 0.422 & $0.709(0.310-1.623)$ & 0.416 \\
Pathological grade & 0.011 & 0.357 & $1.011(0.502-2.035)$ & 0.975 \\
Maximum tumor diameter & -0.127 & 0.509 & $0.881(0.325-2.391)$ & 0.804 \\
Ascites amount & 0.623 & 0.445 & $1.865(0.779-4.464)$ & 0.162 \\
Size of residual tumor & 0.192 & 0.426 & $1.211(0.525-2.793)$ & 0.653 \\
Serum CA125 & 0.165 & 0.403 & $1.180(0.536-2.598)$ & 0.681 \\
HES1 expression & 0.787 & 0.430 & $2.198(0.947-5.102)$ & 0.067 \\
HES5 expression & -0.441 & 0.429 & $0.643(0.278-1.490)$ & 0.303 \\
\hline
\end{tabular}

prognostic factors in early stage cervical carcinoma, including later stage, lymph node metastasis, poor differentiation, larger tumor size $(>2 \mathrm{~cm})$, and deeper cervical invasion (Liu et al. 2007). In this study, we found that the expressions of HES1 and HES5 were significantly higher in ovarian serous adenocarcinoma than those in adenoma and normal control, suggesting that increased expressions of HES1 and HES5 protein exist in ovarian serous cancer and probably contribute to tumor progression and poorer prognosis. The elevated HES1 and HES5 expression were probably induced by Notch activation, but a further study is needed for exploring which Notch paralog is responsible for such induction because one Notch paralog may play different role from other in Hes activation (Beatus et al. 1999).

The association of Notch signaling with response to chemotherapy and prognosis in cancer patients has been relatively well clarified. Nefedova and colleagues found that activation of Notch-1 resulted in protection of myeloma and malignant lymphoid cells from melphalan- and mitoxantrone-induced apoptosis and this protection was associated with up-regulation of p21 (WAF/Cip) and growth inhibition of cells (Nefedova et al. 2004). Similarly, Notch1 protein participated in P53-mediated chemoresistance in breast cancers (Colaluca et al. 2008). A clinical study showed that activating Notch 1 mutations could predict favorable early chemotherapy response and long-term outcome in childhood precursor T-cell lymphoblastic leukemia (Breit et al. 2006). However, the influence of HES genes, essential effectors of Notch signaling, on response to chemotherapeutic drugs was still very rare reported up to date. A recent study in vitro and in mice model by Nefedova and colleagues showed that inhibition of Notch signaling prevented drug resistance and sensitizes myeloma to chemotherapy, and HES1 mediated this procedure (Nefedova et al. 2008). Although we did not find that the patients with different Hes expressions presented different responses to chemotherapy in this study, our clinical follow-up data showed that overall and disease-free survival period of the patients with HES1 low-expression were longer than those with high-expression, especially a significant difference of overall survival existed between
HES1 low-expression and high-expression. This is the first primary clinical investigation on the correlation between HES protein expression and prognosis in ovarian epithelial cancers, to our knowledge. Our findings suggest that the expressions of bHLH gene HES1 and HES5 are increased in advanced ovarian serous adenocarcinomas, and HES1 high-expression probably is a potential poor prognostic factor for the patients. However, our univariate analysis and multivariate Cox regression model did not show that HES1 or HES5 expression was a factor associated with survival of advanced ovarian serous adenocarcinoma patients. It is likely to be due to lack of enough samples and a further study with more cases seems to be needed.

In conclusion, our results suggest that the expressions of bHLH gene HES1 and HES5 are increased in advanced ovarian serous adenocarcinomas, and HES1 high-expression probably is a potential poor prognostic factor for the patients.

Acknowledgments We greatly thank the foundations for their grants to support our study, including Zhejiang Natural Science Foundation (X206961), Zhejiang Province Health Bureau Project (2006QN018), and Zhejiang Province Education Bureau Project (20070133). The funding sources were not involved in the study design, in the collection, analysis, and interpretation of data; in the writing of the manuscript; and in the decision to submit the manuscript for publication.

Conflicts of interest statement There no conflicts of interest to declare.

Open Access This article is distributed under the terms of the Creative Commons Attribution Noncommercial License which permits any noncommercial use, distribution, and reproduction in any medium, provided the original author(s) and source are credited.

\section{References}

Axelson H (2004) The Notch signaling cascade in neuroblastoma: role of the basic helix-loop-helix HASH-1 and HES-1. Cancer Lett 204(2):171-178. doi:10.1016/S0304-3835(03)00453-1

Beatus P, Lundkvist J, Oberg C, Lendahl U (1999) The Notch3 intracellular domain represses notch1-mediated activation 
through hairy/enhancer of split (HES) promoters. Development 126(17):3925-3935

Behboudi A, Enlund F, Winnes M, Andrén Y, Nordkvist A, Leivo I, Flaberg E, Szekely L, Mäkitie A, Grenman R, Mark J, Stenman G (2006) Molecular classification of mucoepidermoid carcinomas-prognostic significance of the MECT1-MAML2 fusion oncogene. Genes Chromosomes Cancer 45(5):470-481. doi: 10.1002/gcc. 20306

Breit S, Stanulla M, Flohr T, Schrappe M, Ludwig WD, Tolle G, Happich M, Muckenthaler MU, Kulozik AE (2006) Activating NOTCH1 mutations predict favorable early treatment response and long-term outcome in childhood precursor T-cell lymphoblastic leukemia. Blood 108(4):1151-1157. doi:10.1182/blood2005-12-4956

Bridgewater JA, Nelstrop AE, Rustin GJ, Gore ME, McGuire WP, Hoskins WJ (1999) Comparison of standard and CA-125 response criteria in patients with epithelial ovarian cancer treated with platinum or paclitaxel. J Clin Oncol 17(2):501-508

Colaluca IN, Tosoni D, Nuciforo P, Senic-Matuglia F, Galimberti V, Viale G, Pece S, Di Fiore PP (2008) NUMB controls p53 tumor suppressor activity. Nature 451(7174):76-80. doi:10.1038/ nature 06412

Fu Y, Ye D, Chen H, Lu W, Ye F, Xie X (2007) Weakened spindle checkpoint with reduced BubR1 expression in paclitaxel-resistant ovarian carcinoma cell line SKOV3-TR30. Gynecol Oncol 105(1):66-73. doi:10.1016/j.ygyno.2006.10.061

Hopfer O, Zwahlen D, Fey M, Aebi S (2005) The Notch pathway in ovarian carcinoma and adenomas. Br J Cancer 93(6):709-718. doi:10.1038/sj.bjc.6602719

Hoskins WJ, Perez CA, Young RC (2000) Epithelial ovarian cancer. In: Jonathan SB (ed) Principles and practice of gynecologic oncology, 4th edn. Lippincott Williams and Wilkins, Ediburgh, pp 1005-1057

Innocenzi D, Alò PL, Balzani A, Sebastiani V, Silipo V, La Torre G, Ricciardi G, Bosman C, Calvieri S (2003) Fatty acid synthase expression in melanoma. J Cutan Pathol 30(1):23-28

Ishimura N, Bronk S, Gores G (2005) Inducible nitric oxide synthase up-regulates Notch-1 in mouse cholangiocytes: implications for carcinogenesis. Gastroenterology 128(5):1354-1368

Ito T, Udaka N, Yazawa T, Okudela K, Hayashi H, Sudo T, Guillemot F, Kageyama R, Kitamura H (2000) Basic helix-loop-helix transcription factors regulate the neuroendocrine differentiation of fetal mouse pulmonary epithelium. Development 127(18): 3913-3921

Jensen J, Pedersen EE, Galante P, Hald J, Heller RS, Ishibashi M, Kageyama R, Guillemot F, Serup P, Madsen OD (2000) Control of endodermal endocrine development by HES-1. Nat Genet 24(1):36-44. doi:10.1038/71657

Kimura K, Satoh K, Kanno A, Hamada S, Hirota M, Endoh M, Masamune A, Shimoseqawa T (2007) Activation of Notch signaling in tumorigenesis of experimental pancreatic cancer induced by dimethylbenzanthracene in mice. Cancer Sci 98(2): $155-162$

Kunnimalaiyaan M, Yan S, Wong F, Zhang YW, Chen H (2005) Hairy enhancer of Split-1 (HES-1), a Notch1 effector, inhibits the growth of carcinoid tumor cells. Surgery 138(6):1137-1142. doi:10.1016/j.surg.2005.05.027
Lee S, Choi EJ, Jin C, Kim DH (2005) Activation of PI3 K/Akt pathway by PTEN reduction and PIK3CA mRNA amplification contributes to cisplatin resistance in an ovarian cancer cell line. Gynecol Oncol 97(1):26-34. doi:10.1016/j.ygyno.2004.11.051

Liu J, Ye F, Chen H, Lu W, Zhou C, Xie X (2007) Expression of differentiation associated protein HES1 and HES5 in cervical squamous carcinoma and its precursors. Int $\mathbf{J}$ Gynecol Cancer 17(6):1293-1299. doi:10.1111/j.1525-1438.2007.00930.x

Miller AB, Hoogstraten B, Staquet M, Winkler A (1981) Reporting results of cancer treatment. Cancer 47(1):207-214

Miyazaki M, Kawamoto H, Kato Y, Itoi M, Miyazaki K, Masuda K, Tashiro S, Ishihara H, Igarashi K, Amagai T, Kanno R, Kanno M (2005) Polycomb group Gene mel-18 regulates early T progenitor expansion by maintaining the expression of HES-1, a target of the Notch Pathway. J Immunol 174(5):2507-2516

Mozzetti S, Ferlini C, Concolino P, Filippetti F, Raspaglio G, Prislei S, Gallo D, Martinelli E, Ranelletti FO, Ferrandina G, Scambia G (2005) Class III beta-tubulin overexpression is a prominent mechanism of paclitaxel resistance in ovarian cancer patients. Clin Cancer Res 11(1):298-305

Mungamuri S, Yang X, Thor A, Somasundaram K (2006) Survival signaling by Notch1: mammalian target of rapamycin (mTOR)dependent inhibition of P53. Cancer Res 66(9):4715-4724. doi:10.1158/0008-5472.CAN-05-3830

Nefedova Y, Cheng P, Alsina M, Dalton WS, Gabrilovich DI (2004) Involvement of Nortch-1 signaling in bone marrow stromamediated de novo drug resistance of myeloma and other malignant lymphoid cell lines. Blood 103(9):3503-3510. doi:10.1182/blood-2003-07-2340

Nefedova Y, Sullivan DM, Bolick SC, Dalton WS, Gabrilovich DI (2008) Inhibition of Notch signaling induces apoptosis of myeloma cells and enhances sensitivity to chemotherapy. Blood 111(4):2220-2229. doi:10.1182/blood-2007-07-102632

Park SJ, Armstrong S, Kim CH, Yu M, Robertson K, Kelley MR, Lee SH (2005) Lack of EGF receptor contributes to drug sensitivity of human germline cells. $\mathrm{Br} \mathrm{J}$ Cancer 92(2):334-341. doi: $10.1038 /$ sj.bjc. 6602315

Politi K, Feirt N, Kitajewski J (2004) Notch in mammary development and breast cancer. Semin Cancer Biol 14(5):341-347. doi: 10.1016/j.semcancer.2004.04.013

Ross DA, Hannenhalli S, Tobias J, Cooch N, Shiekhattar R, Kadesch T (2006) Functional analysis of HES-1 in preadipocytes. Mol Endocrinol 20(3):698-705. doi:10.1210/me.2005-0325

Shi Y, Sun G, Zhao C, Steward S (2008) Neural stem cell selfrenewal. Crit Rev Oncol Hematol 65(1):43-53. doi:10.1016/j. critrevonc.2007.06.004

Ström A, Arai N, Leers J, Gustafsson JA (2000) The hairy and enhancer of split homologue-1 (HES-1) mediates the proliferative effect of 17beta-estradiol on breast cancer cell lines. Oncogene 19(51):5951-5953

Zhou J, Liu M, Luthra R, Jones J, Aneja R, Chandra R, Tekmal RR, Joshi HC (2005) EM012, a microtubule-interfering agent, inhibits the progression of multidrug-resistant human ovarian cancer both in cultured cells and in athymic nude mice. Cancer Chemother Pharmacol 55(5):461-465. doi:10.1007/s00280-0040903-1 